\title{
Rate Allocation in Massive Multiple Access Combining Successive Decoding with Error Control
}

\author{
Francesc Molina and Josep Sala-Álvarez \\ Signal Theory and Communications Department, Technical University of Catalonia (UPC) \\ E-mail: \{francesc.molina, josep.sala $\} @$ upc.edu
}

\begin{abstract}
The Gaussian multiple access channel under asymptotically many code-division-multiple-access users and successive interference cancellation (SIC) is investigated in this work. Users transmit $n$-symbol packets and select their encoding rate as a function of their perfectly known channel gains. We address a theoretical study whereby the performance of employed encoding schemes is described by achievable second-order coding rates at blocklength $n$. Our contribution is twofold. Firstly, we formulate and solve an optimal control problem to maximize the asymptotic user-aggregate spectral efficiency (SE) for a one-iteration SIC. Secondly, we analyze the user-limit behavior of two-iteration SIC and propose a complexity-affordable vector optimization to maximize the user-aggregate SE. Reported results quantify the benefit of adopting a two-iteration SIC versus the blocklength.

Index Terms-Massive multiple access, finite blocklength regime, random spreading, packet error rate.
\end{abstract}

\section{INTRODUCTION}

The rapid growth in the number of simultaneously connected devices has prompted many investigations on the Gaussian multiple access channel in the many-user regime [1]-[3]. Cost-effective physical layer configurations that enable reliable multiple access to a high number of simultaneous transmitters with low coordination among devices are direct-sequence spread spectrum (dense spreading) [4] and low-density spreading [5]. This work concentrates on dense spreading whereby users adopt non-orthogonal waveforms with large spreading gains, and successive interference cancellation (SIC) is used on the receiver side. Practical examples for this physical layer are found in some satellite scenarios e.g. in [6].

For this configuration, it is known that SIC benefits from a severe imbalance between the energy and rate of each user. In the infinite blocklength regime, SIC attains error-free decoding when users' encoding rates are adjusted based on their individual signal-to-noise-plus-interference ratios (SINR) [7]. Information theoretic analyses have shown, however, that the asymptotic-length results are unattainable with short packets since communication is subject to packet errors [8]. Consequently, users may be, eventually, decoded unsuccessfully. In these cases, system performance can be further improved by iterating the multiuser SIC, namely iterative SIC (I-SIC).

This work has been supported by the Spanish Ministry of Science and Innovation through projects WINTER (TEC2016-76409-C2-1-R / AEI / FEDER, UE) and RODIN (PID2019-105717RB-C22 / AEI / 10.13039/501100011033) and by the Catalan Government (AGAUR) under grant 2017 SGR 578. F. Molina is also supported by Fellowship 2020FI_B2 00062 (Secretary for Universities and Research of the Generalitat de Catalunya and the European Social Fund).
Along this line, different I-SIC strategies as a function of the reliability of symbol decisions have been explored. In [9], I-SIC is equipped with a bank of soft-input-softoutput decoders, and cancelations are performed according to symbol reliability. In [6], [10], a cyclic redundancy check is encapsulated next to data, and I-SIC operates at each iteration only over users unsuccessfully decoded in previous iterations. Both latter studies are limited to the case when users share the same encoder where, as shown in [10], the optimal energy distribution that maximizes SIC's performance is exponential.

In this work, we fill the gap in the previous literature by considering many encoders and by deriving the optimal rate allocation for asymptotically many users. Our study carries out a progressive analysis from the ideal to the more practical SIC policies. For one-iteration SIC, we find the optimal rate allocation by solving an optimal control problem. For the two-iteration SIC, we design the rate allocation scheme by solving a vector optimization problem derived from our userasymptotic analysis.

With regard to paper notation: The dot notation is used for univariate differentiation: $\dot{g}(x)$ is the derivative of $g(x)$. Partial derivatives of $G(x, y)$ are indicated by $G_{x}$ and $G_{y}$.

\section{Problem Statement}

We consider a massive access setting where $K$ code-division multiple access users transmit $n$-symbol packets with average symbol energy $\bar{E}$. User $k$ adopts a physical layer composed of a coded modulation scheme, that allows for transmission at rate $R[k]$, plus a spreading code, with spreading gain $N$, taken as part of a long pseudonoise sequence.

Let $h[k]$ be the perfectly known channel power gain for user $k$ used to adapt its transmission rate $R[k]$ as

$$
R[k]=f(h[k]),
$$

where $f(h)$ is the allocation function designed in this work so as to maximize a network-centric utility. More concretely, the goal of this work is the determination of the $R[1], \ldots, R[K]$ that maximize the user-aggregate spectral efficiency

$$
\mathrm{SE}=\frac{1}{N} \sum_{k=1}^{K} R[k] \cdot(1-\operatorname{per}[k])
$$

when imperfect SIC operates at reception to decode all users. In expression (2), per $[k]$ stands for the packet error rate associated with user $k$, introduced to model practical effects of decoding short packets. 
If $K$ is sufficiently large, we may turn the previous expression to the asymptotic spectral efficiency (ASE) key performance indicator defined by (i) the asymptotic relationship $\alpha=K / N$; and (ii) the user-index $t=\lim _{K \rightarrow+\infty} k / K$, as

$$
\mathrm{ASE}=\alpha \int_{0}^{1} R(t) \cdot(1-\operatorname{per}(t)) \mathrm{d} t
$$

where the function sought is the rate allocation profile $R(t)$ in $0 \leq t \leq 1$ rather than the finite set $R[1], \ldots, R[K]$.

Naturally, the explicit form for $\operatorname{per}(t)$, or its impact on the ASE utility may depend on the adopted SIC decodingcancellation system implementation. The following sections are devoted: firstly, to explaining briefly a system model for each policy; and secondly, to computing the sought allocation.

\section{System Analysis And Rate Allocation FOR ONE-ITERATION SIC}

In this section, we address the design of optimal rate allocation schemes for two SIC approaches that contemplate only one decoding attempt per user (one-iteration SIC). We carry out the analysis of such SIC policies varying from less to more practical operative schemes. Both SIC strategies share the same core implementation, which we summarize next:

(i) Firstly, users $1 \leq k \leq K$ are ranked in non-increasing order of user-channel gains, coincident with the order of received symbol energies

$$
E_{\mathrm{r}}[1] \geq \cdots \geq E_{\mathrm{r}}[k] \geq \cdots \geq E_{\mathrm{r}}[K]
$$

$E_{\mathrm{r}}[k]=\bar{E} \cdot h[k]$ is the received symbol energy for user $k$ in a quasi-static fading channel.

(ii) Secondly, users are sequentially decoded under the singleuser approach. That is, treating interference as extra noise added to thermal noise. After decoding, we enable error control at reception by enforcing users to generate and encode a CRC jointly with data.

(iii) Thirdly, user cancellation is only produced when the corresponding $\mathrm{CRC}$ checks out. Otherwise, no cancellation occurs.

The subsequent analyses follow the same structure. We first derive a simple system model to analyze the evolution of the interference level as SIC progresses, and later on, we use it to derive the optimal allocation designs.

\section{A. Allocation Design with Optimal First-Order Rates}

We first analyze the ideal case in which users employ capacity-achieving encoding systems (optimal first-order rates) with $n \rightarrow+\infty$, and the receiver operates with perfect cancellation. In this case, the receiver is able to decode all users without error as long as the SINR of each user satisfies

$$
\Gamma[k]=\frac{E_{\mathrm{r}}[k]}{N_{t}[k]} \geq b^{R[k]}-1 .
$$

$b$ is the base used to measure rates. For this SIC system implementation, the noise plus interference term for user decoded at stage $k$ is

$$
N_{t}[k]=N_{0}+\frac{\theta}{N} \sum_{i>k} E_{\mathrm{r}}[i] .
$$

$N_{0}$ is the noise power spectral density, and $\theta / N$ is the second moment of the cross-correlation between different spreading signatures, with $0 \leq \theta \leq 1$ the linear factor modelling time variations with respect to the synchronous case.

In the user-limit case, the summation can be turned to an integral and thus, the noise plus interference profile reads

$$
N_{t}(t)=N_{0}+\alpha \theta \int_{0}^{t} E_{\mathrm{r}}(\tau) \mathrm{d} \tau .
$$

Since users employ capacity-achieving encodings, they must adapt their transmission rates according to their SINRs $\Gamma[k]=$ $E_{\mathrm{r}}[k] / N_{t}[k] \rightarrow \Gamma(t)=E_{\mathrm{r}}(t) / N_{t}(t)$ as (8), and thus, the maximum attainable network performance is given by (9)

$$
\begin{aligned}
R(t) & =\log _{b}\left(1+\frac{E_{\mathrm{r}}(t)}{N_{t}(t)}\right) \quad \text { in } \quad 0 \leq t \leq 1 \\
\mathrm{ASE} & =\alpha \int_{0}^{1} \log _{b}\left(1+\frac{E_{\mathrm{r}}(t)}{N_{t}(t)}\right) \mathrm{d} t
\end{aligned}
$$

\section{B. Allocation Design with Optimal Second-Order Rates}

We now consider a more functional SIC that operates under two practical features:

1) Non-ideal decoding: Due to the transmission of (short) packets of $n$ symbols, the receiver may decode some users unsuccessfully. In this case, we may adopt, instead of capacityachieving encoders, encoding schemes that achieve optimal second-order rates at blocklength $n$. We consider, for each encoding system of rate $R$, the known packet error rate versus SINR curve PER $[\Gamma, R]$. In this respect, the analytical findings in [8] provide the following relationship between rate $R$, communication error per and blocklength $n$

$$
R=\log (1+\Gamma)-\sqrt{\frac{V}{n}} \cdot Q^{-1}(\text { per })+\frac{\log n}{n},
$$

with $V \triangleq\left(1-(1+\Gamma)^{2}\right) \log _{b}(e)$ and $Q(\cdot)$ the Q-function. By allowing free variations on the SINR $\Gamma$ and the rate $R$, an analytic form to the bivariate packet error rate curve with arguments $\Gamma$ and $R$ can be obtained as

$$
\operatorname{PER}[\Gamma, R]=Q\left[\sqrt{\frac{n}{V}}\left(\log (1+\Gamma)-R+\frac{\log n}{n}\right)\right] .
$$

2) Imperfect cancellation: We consider that the signal of each user is not regenerated perfectly, and thus, a fraction of its energy remains uncanceled. We adopt a general cancellation model that depends on the SINR and on the employed decoder, in which the known function $\varepsilon[\Gamma, R]$ models the remaining fraction of energy conditioned on successful decoding.

Our system model for this case should distinguish between successfully and unsuccessfully decoded users. To that aim, we check out the CRC after channel decoding to determine packet decoding errors, whose binary decision is incorporated to our system model by means of the random variable $\epsilon[k]$ for the decoding-cancellation operation of user $k$. In agreement with [10], we consider unsuccessful decoding $(\epsilon[k]=1)$ with probability $\operatorname{PER}[\Gamma[k], R[k]]$ and successful decoding $(\epsilon[k]=\varepsilon[\Gamma[k], R[k]])$ with probability $\operatorname{PSR}[\Gamma[k], R[k]]$ (PSR is the complementary PER function). 
The $k$-th user SINR is $\Gamma[k]=E_{\mathrm{r}}[k] / N_{t}[k]$ with the noise plus interference term associatd with user $k$ computed as

$$
N_{t}[k]=N_{0}+\frac{\theta}{N} \sum_{i<k} \epsilon[i] E_{\mathrm{r}}[i]+\frac{\theta}{N} \sum_{i>k} E_{\mathrm{r}}[i] .
$$

The second term is the interference generated by already processed users, more concretely, the (successfully decoded) imperfectly canceled users plus those unsuccessfully decoded. The third term corresponds to users not processed yet.

In the user-limit case, we proceed likewise as we did in the previous section in order to obtain

$$
N_{t}(t)=N_{0}+\alpha \theta \int_{0}^{t} \epsilon(\tau) E_{\mathrm{r}}(\tau) \mathrm{d} \tau+\alpha \theta \int_{t}^{1} E_{\mathrm{r}}(\tau) \mathrm{d} \tau,
$$

where, invoking the Kolmogorov's strong law of large numbers, the random variable $\epsilon(\tau)$ can be substituted by its average $\mathbb{E}[\epsilon]=1-\Phi[\Gamma, R]$ and $\Phi[\Gamma, R]=(1-\varepsilon[\Gamma, R]) \Gamma \cdot \operatorname{PSR}[\Gamma, R]$.

Equation (13) can be simplified by turning it to a dynamic equation. This is possible by computing the term $\dot{N}_{t}(t) / N_{t}(t)$, which reads

$$
\frac{\dot{N}_{t}(t)}{N_{t}(t)}=-\alpha \cdot \Phi\left[\frac{E_{\mathrm{r}}(t)}{N_{t}(t)}, R(t)\right]
$$

and that describes the evolution of the noise plus interference level as SIC progresses by means of an ordinary differential equation with the initial value

$$
N_{t}(0)=N_{0}+\alpha \theta \int_{0}^{1} E_{\mathrm{r}}(t) \mathrm{d} t .
$$

We design the rate allocation in the user-asymptotic case by solving the following optimal control problem. The profit is ASE and the state variable is $N_{t}(t)$ :

$$
\begin{aligned}
& \max _{R(t)} \alpha \int_{0}^{1} R(t) \cdot \operatorname{PSR}\left[\frac{E_{\mathrm{r}}(t)}{N_{t}(t)}, R(t)\right] \mathrm{d} t \\
& \text { s.t. eqs. (14)-(15) }
\end{aligned}
$$

We follow standard optimization tools in optimal control theory in form of the Hamiltonian formulation [11]. For simplicity, functions of the variable $t$ are indicated in boldface. Then, the Hamiltonian reads

$$
\boldsymbol{H} \triangleq \boldsymbol{R} \cdot \operatorname{PSR}\left[\frac{\boldsymbol{E}_{\mathrm{r}}}{\boldsymbol{N}_{t}}, \boldsymbol{R}\right]-\boldsymbol{\lambda} \cdot \Phi\left[\frac{\boldsymbol{E}_{\mathrm{r}}}{\boldsymbol{N}_{t}}, \boldsymbol{R}\right] .
$$

Therefore, the optimal behavior is attained when the derivative of the Hamiltonian with respect to the sought function vanishes: $\partial \boldsymbol{H} / \partial \boldsymbol{R}=0$. This gives the following non-linear equation for each user $0 \leq t \leq 1$

$$
\lambda \Phi_{R}\left[\frac{\boldsymbol{E}_{\mathrm{r}}}{\boldsymbol{N}_{t}}, \boldsymbol{R}\right]=\boldsymbol{R} \cdot \mathrm{PSR}_{R}\left[\frac{\boldsymbol{E}_{\mathrm{r}}}{\boldsymbol{N}_{t}}, \boldsymbol{R}\right]+\mathrm{PSR}\left[\frac{\boldsymbol{E}_{\mathrm{r}}}{\boldsymbol{N}_{t}}, \boldsymbol{R}\right]
$$

with variable $\lambda$, computed as $\dot{\lambda}=-\alpha N_{t} \frac{\partial H}{\partial N_{t}}$, satisfying

$$
\dot{\boldsymbol{\lambda}}=\alpha \boldsymbol{R} \operatorname{PSR}_{\Gamma}\left[\frac{\boldsymbol{E}_{\mathrm{r}}}{\boldsymbol{N}_{t}}, \boldsymbol{R}\right] \frac{\boldsymbol{E}_{\mathrm{r}}}{\boldsymbol{N}_{t}}-\alpha \boldsymbol{\lambda} \Phi_{\Gamma}\left[\frac{\boldsymbol{E}_{\mathrm{r}}}{\boldsymbol{N}_{t}}, \boldsymbol{R}\right] \frac{\boldsymbol{E}_{\mathrm{r}}}{\boldsymbol{N}_{t}}
$$

with the boundary $\lambda(1)=0$.
Numerical implementation: Since the derived equations (18)-(19) does not provide $R(t)$ in explicit form, we address a numerical algorithm to obtain it. In particular, we sample $t \in[0,1]$ in $K$ uniform intervals and solve (18) for each $t_{i}$, after which, $\lambda\left(t_{i+1}\right)$ is updated as $\lambda\left(t_{i+1}\right)=\lambda\left(t_{i}\right)+\dot{\lambda}\left(t_{i}\right) \frac{1}{K}$. $\lambda(0)$ is found by means of bisection search.

\section{System Analysis and Rate Allocation for TWO-ITERATION SIC}

In this section, we analyze the two-iteration SIC which allows for the improvement of previous results by running a new SIC iteration only over users that survive unsuccessfully decoded at the end of the first SIC iteration.

\section{A. System Model for the First SIC Iteration}

For the first SIC iteration, we consider the same system model we propose in Section III-B for the one-iteration SIC. In this section, we include the superindex 1 to the SINRs and noise plus interference terms to make explicit reference to the first SIC iteration. We have $\Gamma^{1}[k]=E_{\mathrm{r}}[k] / N_{t}^{1}[k]$ with

$$
N_{t}^{1}[k]=N_{0}+\frac{\theta}{N} \sum_{i<k} \epsilon[i] E_{\mathrm{r}}[i]+\frac{\theta}{N} \sum_{i>k} E_{\mathrm{r}}[i] .
$$

Recall that $\epsilon[i]$ is the random variable that captures decoding and imperfect cancellation as defined in Section III-B.

At the end of the first SIC iteration, we have an equivalent interference term equal to the noise power spectral density plus the interference from users canceled imperfectly throughout the first SIC iteration

$$
N_{0}^{\prime}=N_{0}+\frac{\theta}{N} \sum_{i=1}^{K} \epsilon^{\prime}[i] E_{\mathrm{r}}[i] .
$$

$\epsilon^{\prime}[i]$ equals 0 when the CRC does not check out and $\varepsilon\left[\Gamma^{1}[i], R[i]\right]$ when the $i$-th user is recovered successfully.

\section{B. System Model for the Second SIC Iteration}

We consider that after the first SIC iteration $K_{2}$ users survive unsuccessfully decoded. Then, the second SIC iteration handles $K_{2}$ users, that we index as $1 \leq k_{2} \leq K_{2}$. We discuss a very useful system model for this SIC iteration in the sequel. In the same way as in [10], we define the following relationship between $k$, used for the first SIC iteration, and $k_{2}$, used for the second SIC iteration, as [10]

$$
\phi: k \longleftrightarrow k_{2}
$$

which allows to determine the index of user $k$ in the second SIC iteration as $\phi[k]$, and vice-versa, to obtain the index of user $k_{2}$ in the initial ordering as $\phi^{-1}\left[k_{2}\right]$.

A relevant aspect we may discuss for this iteration is the behavior of channel decoding. Clearly, the success of channel decoding depends on whether the noise plus interference signal affecting user $\phi\left[k_{2}\right]$ in the first iteration has changed for the same user $k_{2}$ in the second SIC iteration. We take up the proposal in [10] for a single encoder, and we extend it to multiple encoders by adopting, for the encoder of rate $R$, the multivariate PER PER $2\left[\Gamma^{1}, \Gamma^{2}, R\right]$ with the arguments $\Gamma^{1}$ and 
$\Gamma^{2}$ denoting the SINRs experienced by a user in each iteration. Due to computational difficulties in obtaining such a function, we approximate it by the expression in [10, eq. (26)]

$$
\operatorname{PER}_{2}\left[\Gamma^{1}, \Gamma^{2}, R\right] \approx \frac{\operatorname{PER}\left[\Gamma^{2}, R\right]}{\operatorname{PER}\left[\Gamma^{1}, R\right]} .
$$

Then, the SINR for user $k_{2}$ is $\Gamma^{2}\left[k_{2}\right]=E_{\mathrm{r}}\left[k_{2}\right] / N_{t}^{2}\left[k_{2}\right]$ with the noise plus interference term computed as

$$
\begin{aligned}
N_{t}^{2}\left[k_{2}\right] & =N_{0}^{\prime} \\
& +\frac{\theta}{N} \sum_{i<k_{2}} \epsilon_{2}[i] E_{\mathrm{r}}\left[\phi^{-1}[i]\right]+\frac{\theta}{N} \sum_{i>k_{2}} E_{\mathrm{r}}\left[\phi^{-1}[i]\right],
\end{aligned}
$$

with $\epsilon_{2}[i]$ equal to 1 when the CRC does not check out, with probability $\operatorname{PER}_{2}\left[\Gamma^{1}\left[\phi^{-1}[i]\right], \Gamma^{2}[i], R[i]\right]$, and equal to $\varepsilon\left[\Gamma^{2}[i], R[i]\right]$ with complementary probability.

\section{User-Limit Analysis of the First SIC iteration}

The user-limit analysis of the first SIC iteration concludes identically as in Section III-B, where: firstly, the noise plus interference term evolves as (14); secondly, the average number of users decoded unsuccessfully is computed as

$$
\overline{\operatorname{per}}_{1}=\int_{0}^{1} \operatorname{PER}\left[\frac{E_{\mathrm{r}}(t)}{N_{t}^{1}(t)}, R(t)\right] \mathrm{d} t ;
$$

and thirdly, the second SIC iteration starts with an equivalent noise plus interference term (21) that now reads

$$
\begin{aligned}
N_{0}^{\prime} & =N_{0} \\
& +\alpha \theta \int_{0}^{1} E_{\mathrm{r}}(t) \varepsilon\left[\frac{E_{\mathrm{r}}(t)}{N_{t}^{1}(t)}, R(t)\right] \operatorname{PSR}\left[\frac{E_{\mathrm{r}}(t)}{N_{t}^{1}(t)}, R(t)\right] \mathrm{d} t .
\end{aligned}
$$

\section{User-Limit Analysis of the Second SIC iteration}

In the second SIC iteration we consider that a sufficiently high number of users is present so that the traffic load relationship for this iteration is asymptotically given by

$$
\alpha_{2}=K_{2} / N \longrightarrow \alpha \overline{\operatorname{per}}_{1} .
$$

Moreover, the user-index becomes

$$
t_{2}=\lim _{K_{2} \rightarrow+\infty} k_{2} / K_{2},
$$

and that can be easily related with the first user-index $t$ recalling that, in the user-limit case, each interval $\mathrm{d} t$ leaves a fraction $\operatorname{PER}\left[\Gamma^{1}(t), R(t)\right] \mathrm{d} t$ of users unsuccessfully decoded. As stated in [10], this results in the relationship

$$
t_{2}=\phi(0 \leq t \leq 1)=\frac{1}{\overline{\operatorname{per}}_{1}} \int_{0}^{t} \operatorname{PER}\left[\frac{E_{\mathrm{r}}(t)}{N_{t}^{1}(t)}, R(t)\right] \mathrm{d} t .
$$

The noise plus interference term (24a) can be turned to an asymptotic quantity if we multiply and divide each sum by $K_{2}$, we introduce the relationship $\alpha_{2}=K_{2} / N$, and we turn summations to integrals with $\mathrm{d} \tau=\lim _{K_{2} \rightarrow \infty} 1 / K_{2}$. Then,

$$
\begin{aligned}
N_{t}^{2}\left(t_{2}\right) & =N_{0}^{\prime}+\alpha_{2} \theta \int_{0}^{t_{2}} \epsilon_{2}(\tau) E_{\mathrm{r}}\left(\phi^{-1}(\tau)\right) \mathrm{d} \tau \\
& +\alpha_{2} \theta \int_{t_{2}}^{1} E_{\mathrm{r}}\left(\phi^{-1}(\tau)\right) \mathrm{d} \tau .
\end{aligned}
$$

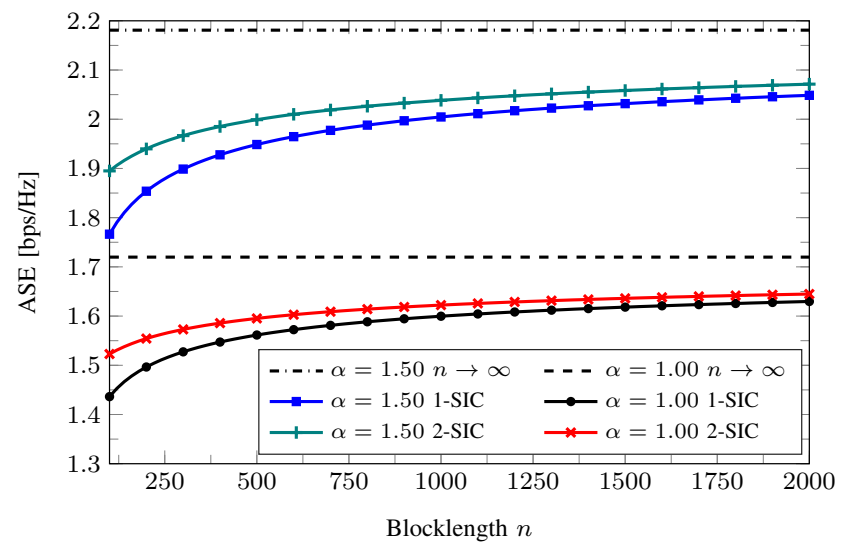

Fig. 1. Asymptotic user-aggregate SE versus blocklength $n$.

We seek, in the same way we did for the first SIC iteration, a differential equation that describes the evolution of the noise plus interference term as SIC mitigates interference. Then, substituting $t_{2}=\phi(t)$ and computing the term $\dot{N}_{t}^{2}(\phi(t))=$ $\frac{\mathrm{d}}{\mathrm{d} t} N_{t}^{2}(\phi(t))$ we get the differential equation

$$
\frac{\dot{N}_{t}^{2}(\phi(t))}{N_{t}^{2}(\phi(t))}=-\alpha \cdot \Psi\left[\frac{E_{\mathrm{r}}(t)}{N_{t}^{1}(t)}, \frac{E_{\mathrm{r}}(t)}{N_{t}^{2}(\phi(t))}, R(t)\right] .
$$

$\Psi\left[\Gamma^{1}, \Gamma^{2}, R\right] \triangleq \theta\left(1-\varepsilon\left[\Gamma^{2}, R\right]\right)\left(\operatorname{PER}\left[\Gamma^{1}, R\right]-\operatorname{PER}\left[\Gamma^{2}, R\right]\right)$, and the initial value is $N_{t}^{2}(0)=N_{t}^{1}(1)$, as

$$
N_{t}^{2}(0)=N_{t}^{1}(0) \exp \left(-\alpha \int_{0}^{1} \Phi\left[\frac{E_{\mathrm{r}}(t)}{N_{t}^{1}(t)}\right] \mathrm{d} t\right) .
$$

\section{E. Rate Allocation Design}

The following optimization needs to be solved:

$$
\begin{gathered}
\max _{R(t)} \alpha \int_{0}^{1} R(t) \operatorname{PSR}\left[\frac{E_{\mathrm{r}}(t)}{N_{t}^{2}(\phi(t))}, R(t)\right] \mathrm{d} t \\
\text { s.t. eqs. (14)-(15), (31)-(32) }
\end{gathered}
$$

Indeed, the problem can be solved using optimal control tools, but the resolution of the equations obtained leads to an exhaustive and computationally demanding procedure. Rather, we address (33a)-(33b) by turning: firstly, integrals to Riemann sums by discretizing $t$ as $t_{1}, \ldots, t_{K}$; and secondly, differential equations to finite difference equations. Then, we address the following $K$-multivariate optimization:

$$
\begin{aligned}
& \max _{R\left(t_{1}\right), \ldots, R\left(t_{K}\right)} \frac{\alpha}{K} \sum_{i=1}^{K} R\left(t_{i}\right) \operatorname{PSR}\left[\frac{E_{\mathrm{r}}\left(t_{i}\right)}{N_{t}^{2}\left(\phi\left(t_{i}\right)\right)}, R\left(t_{i}\right)\right] \\
& \text { s.t. } N_{t}^{1}\left(t_{1}\right)=N_{0}+\frac{\alpha \theta}{K} \sum_{i=1}^{K} E_{\mathrm{r}}\left(t_{i}\right) \\
& \text { s.t. } N_{t}^{1}\left(t_{i+1}\right)=N_{t}^{1}\left(t_{i}\right)\left(1-\left.\frac{\alpha}{K} \Phi\right|_{t=t_{i}}\right) \\
& \text { s.t. } N_{t}^{2}\left(\phi\left(t_{1}\right)\right)=N_{t}^{1}\left(t_{M}\right) \\
& \text { s.t. } N_{t}^{2}\left(\phi\left(t_{i+1}\right)\right)=N_{t}^{2}\left(\phi\left(t_{i}\right)\right)\left(1-\left.\frac{\alpha}{K} \Psi\right|_{t=t_{i}}\right)
\end{aligned}
$$




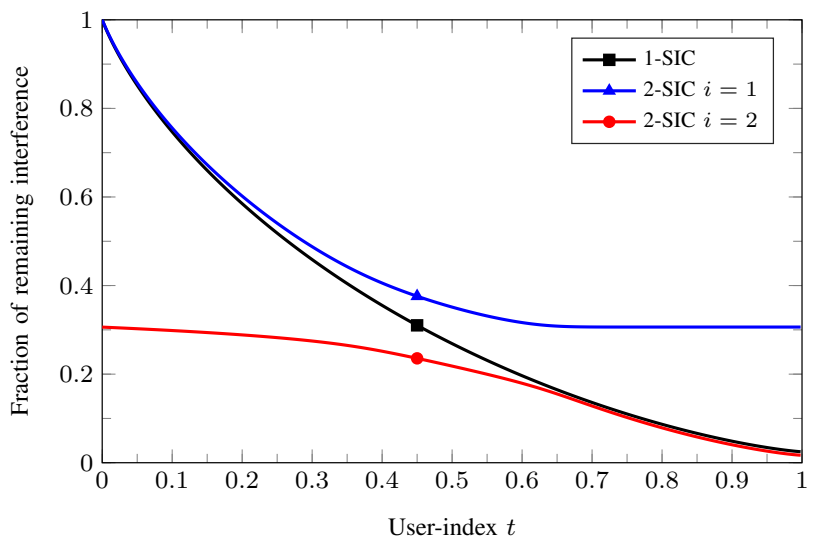

Fig. 2. Fraction of remaining interference as SIC progresses at the traffic load $\alpha=1.50$ and blocklength $n=100$.

\section{Numerical ANALYSis}

We simulate $K=200$ users transmitting packets under the average symbol energy $\bar{E}=10$ and affected by log-normal shadowing. Specifically, the channel gains of all users are obtained from a log-normal distribution with unit mean and deviation 3dB. At the SIC receiver, the uncanceled energy fraction is $\varepsilon=0.01$, and the decorrelation gain $\theta$ is set to 1 .

We draw in Fig. 1 the optimized ASE versus the blocklength $n$. Dashed and dash-dotted lines indicate the optimization in the infinite blocklength regime where, effectively, one-iteration SIC (1-SIC) decodes all users without errors. In the finite blocklength regime, 1-SIC does not suffice, and high ASE loss is experienced for $n \leq 2000$ because the channel coding rate is heavily degraded at low blocklengths [8]. The adopted two-iteration SIC (2-SIC) allows for a slight SE improvement at high blocklengths, and a substantial enhancement of ASE at very low blocklengths, specially so in high activity load situations. The reason is that at high $\alpha$ more interference remains decoded unsuccessfully and imperfectly canceled.

Next, we delve into the operation of the optimal 2-SIC relative to the 1-SIC analyzed previously for an example with traffic load $\alpha=1.50$ and blocklength $n=100$. We draw in Fig. 2 the remaining fraction of interference as SIC progresses with variable $t$. When using 1-SIC, since users are subject only to one decoding attempt, rate allocation must guarantee (statistically) their successful decoding, whereby users are allocated with transmission rates that guarantee individual error rates in $\left[10^{-3}, 10^{-1}\right]$. As shown in Fig. 3, we obtain a more conservative rate allocation relative to that in the infinite blocklength case. When a two-iteration SIC is employed, users can increase slightly their transmission rate in favour of the second decoding attempt. As shown in Fig. 2 , the first SIC iteration (triangle) removes the $70 \%$ of interference by allocating stronger users with rates that guarantee communication errors in the range $\left[10^{-2}, 1\right]$, whereas the last users are allocated with error rates near 1 . This configuration allows for a second decoding attempt (circle) that starts with the $30 \%$ of the initial interference, and enables practically reliable communication for stronger users $\left(\right.$ per $<10^{-6}$ ) to

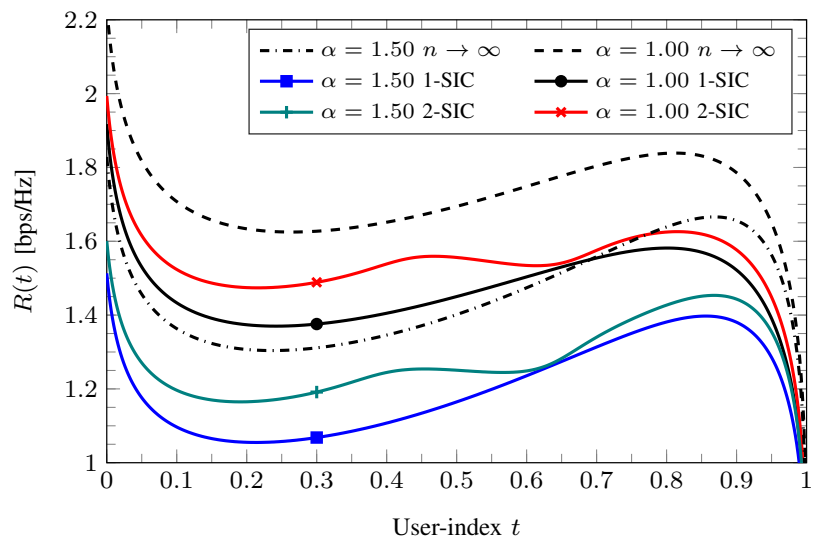

Fig. 3. Per-user encoding rate $R(t)$ in $\mathrm{bps} / \mathrm{Hz}$ versus the user-index $t$ at the blocklength $n=100$.

the detriment of the performance for the last users (weakest) that experience communication error rates near 0.05 .

\section{CONCLUSION}

We have shown that in a massive multiple access network under users employing code-division-multiple-access, successive interference cancellation can be satisfactorily combined with error control to iterate the multiuser receiver over users still undecoded. Rate allocation under one-iteration SIC is undertaken in the user-asymptotic case by leveraging optimal control theory. For two-iteration SIC, the rate allocation problem is solved by means of a vector optimization scheme featuring a very high performance/complexity ratio. More benefits are reported from adopting two-iteration SIC in high traffic load situations, or at very low blocklengths.

\section{REFERENCES}

[1] E. MolavianJazi and J. N. Laneman, "A second-order achievable rate region for gaussian multi-access channels via a central limit theorem for functions," IEEE Trans. Info. Theory, vol. 61, no. 12, pp. 6719-6733, 2015.

[2] E. MolavianJazi and J. N. Laneman, "A finite-blocklength perspective on gaussian multi-access channels," arXiv preprint arXiv:1309.2343, 2013.

[3] Y. Polyanskiy, "A perspective on massive random-access," in 2017 IEEE Int. Symposium on Information Theory (ISIT), 2017, pp. 2523-2527.

[4] S. Verdú, Multiuser detection. Cambridge University Press, 1998.

[5] M. T. P. Le, G. C. Ferrante, T. Q. S. Quek, and M. Di Benedetto, "Fundamental limits of low-density spreading NOMA with fading," IEEE Trans. Wireless Commun., vol. 17, no. 7, pp. 4648-4659, 2018.

[6] F. Collard and R. De Gaudenzi, "On the optimum packet power distribution for spread ALOHA packet detectors with iterative successive interference cancelation," IEEE Trans. Wireless Commun., vol. 13, no. 12, pp. 6783-6794, 2014.

[7] D. V. Djonin and V. K. Bhargava, "Asymptotic analysis of the conventional decision feedback receiver in fading channels," IEEE Trans. Wireless Commun., vol. 2, no. 5, pp. 1066-1078, Sep. 2003.

[8] Y. Polyanskiy, H. V. Poor, and S. Verdú, "Channel coding rate in the finite blocklength regime," IEEE Trans. Inf. Theory, vol. 56, no. 5, pp. 2307-2359, May 2010.

[9] P. S. Rossi, K. Kansanen, R. R. Müller, and C. Rachinger, "Power randomization for iterative detection over random-access fading channels," IEEE Trans. Wireless Commun., vol. 14, no. 10, pp. 5704-5713, 2015.

[10] F. Molina and J. Sala-Álvarez, "Average PER performance metrics of iterative successive interference cancellation," IEEE Wireless Commun. Lett., vol. 9, no. 1, pp. 74-77, 2020.

[11] I. M. Gelfand, R. A. Silverman et al., Calculus of variations. Courier Corporation, 2000. 